El Ángulo del Investigador

\section{Un espacio de aprendizaje para quien comienza a investigar}

Olga Arguedas-Arguedas

Desde hace varios años, el Comité Editorial de Acta Médica Costarricense, ha observado con preocupación el comportamiento de las producciones científicas que los colegas nos envían a la revista para ser publicadas. Como parte de este material, identificamos un grupo de trabajos, usualmente realizados por investigadores noveles, que reflejan una carencia de conocimientos básicos referentes no sólo a qué y cómo investigar, sino también, a la forma de trabajar con los datos y de producir posteriormente un manuscrito científico de calidad. Por lo antes expuesto, y con la esperanza de servir de orientación a todo aquel que se inicia en el campo de la investigación biomédica, hemos decidido la creación de este espacio. No pretendemos hacer una revisión exhaustiva y profunda de todos los temas relacionados con este tópico, pues para tales efectos, existen maravillosos libros de texto, artículos en publicaciones periódicas y cursos de excelente calidad en las universidades. Nuestro objetivo es el de presentar de una manera didáctica los elementos fundamentales para despegar en el espacio de la investigación biomédica. La ruta de vuelo, y el destino final, dependerán del talento, la orientación y la constancia de cada uno.

En nuestros siguientes artículos incluiremos aspectos relacionados con la importancia de la investigación descriptiva, posibilidades y limitaciones de los estudios de investigación según diseño, consejos prácticos para la elaboración de un protocolo de investigación, regulaciones y elementos bioéticos de los diferentes tipos de estudios, conceptos de medicina basada en evidencia para investigadores, guías para la elaboración de un manuscrito científico, entre otros. Asimismo al final de cada entrega, pondremos a disposición de nuestros lectores, un pequeño espacio de "Formule su pregunta", en que el estaremos respondiendo a una consulta enviada por ustedes e ilustrativa de cada tema. Esperamos con optimismo, que esta iniciativa resulte útil.

\section{¿Por qué es importante investigar?}

Aunque resulta una fuerte aseveración, podemos afirmar con bastante certeza, que el profesional de las ciencias de la salud, que no investiga y que no enseña, evoluciona inexorablemente hacia la anquilosis del conocimiento. Si revisamos la historia de la medicina, vemos que esto ha sido así desde el origen de la misma. Los grandes médicos han sido siempre, maestros e investigadores. Paradójicamente las destrezas para investigar y enseñar, no son formalmente desarrolladas en los currículos de formación médica y se aprenden casi siempre por azar o por necesidad.

En nuestro país, el requisito de producir un estudio de investigación está presente en la mayoría de los programas de posgrado en especialidades médicas, evidentemente con la buena intención de introducir al profesional en el campo de la investigación biomédica. Sin embargo, las exigencias laborales y académicas de las residencias y la poca disponibilidad de tutores con sólida formación en investigación, hacen que muchas veces, en vez de generarse una introducción a la investigación "como afición", se cumple con la producción de un trabajo científico aislado y "por obligación". La investigación que se origina sin emoción, con el propósito de llenar un requisito académico, tiene poco sentido. 
Los buenos estudios de investigación, se originan de las dudas o inquietudes que les surgen a personas curiosas, intuitivas e interesadas por ayudar a los demás. La curiosidad médica genera escepticismo, y éste es el motor para comenzar una investigación. El investigador debe partir del hecho de que lo aprendido de otros, puede ser en algunos casos, una verdad provisional. La insatisfacción del médico, es uno de los pilares de donde nace la necesidad de investigar. La inconformidad respecto a los conocimientos vigentes de las enfermedades que afectan a sus pacientes o a su comunidad, o acerca de resultados diagnósticos o terapéuticos, genera la decisión de llevar los problemas de los pacientes al laboratorio, o de reunirlos para valorarlos en conjunto, o de organizarlos para compararlos con casuísticas de otras latitudes. En nuestro sistema de salud, sabemos que la práctica médica revela cotidianamente en forma generosa y seguramente, inagotable, lagunas en el conocimiento, incongruencias, peculiaridades y eventos inesperados, los cuales se constituyen en incentivos primarios para investigar.

El desarrollo de cualquier investigación biomédica, debe tener siempre como norte un propósito beneficente. Desde luego, es agradable el reconocimiento que la comunidad profesional hace a aquel que investiga y publica, sin embargo la importancia de investigar no está en esto, sino en el beneficio que genera en la salud de las personas tanto el conocimiento obtenido como las redes de apoyo nacionales e internacionales que pueden construirse a partir de un trabajo científico. 